\section{Promoting a Culture of Safety}

Over the past 10 years, checklists have been incorporated into the perioperative environment in increasing numbers. The concept appears to be a simple one: develop a simple list of critical items that need to be double-checked before, and in some cases after, any anesthetic or surgery. This has been extended to other phases of perioperative care such as patient handoffs to the PACU (Post-Anesthesia Care Unit) and placement of central lines, for instance. The World Health Organization's "Safe Surgery Saves Lives"1 may be the best known of these instruments. Atul Gawande ${ }^{2}$ popularized checklists in his book, A Checklist Manifesto. Gawande distinguishes between errors of ignorance (mistakes we make because we don't know enough) and errors of ineptitude (mistakes we make because we don't make proper use of what we do know). Mishaps in medicine and many other fields, he writes, are really about the second of these errors, which checklists hope to address.

For those of us who practice in the hospital or ambulatory surgery center (ASC), these checklists have become standard fare. We ensure that the correct patient is in the operating room; the surgical site has been marked to prevent wrong-site surgery; all allergies have been noted; correct antibiotics have been administered; airway concerns, fire risk, and blood loss are discussed; etc. Additionally, all personnel in the operating room introduce themselves and the need for any team member to bring up any issue of concern is reemphasized.

Many of the readers of Anesthesia Progress provide all of their care within the office setting and may not yet fully, or even partially, use checklists. Certainly, some aspects of the checklist procedure may not be as critical in dentistry. In the office setting, we are not as likely to mix up who is getting a sedation or anesthetic and what the surgery is going to be because we tend to know our patients more personally and have more control over clinical care. However, in a busy practice where multiple sedations/general anesthetics (GAs) are provided in a day, it could conceivably happen. Certainly, many dental surgical procedures do not have an indication for perioperative antibiotics, but ensuring that those patients that need them do get them is prudent. Making sure that the right teeth are treated or extracted is still a good thing to do. A brief review of salient medical history concerns might lead to a further discussion of anesthetic, surgical, or postoperative issues, particularly if the surgeon is working with an independent anesthesia provider.

Anesth Prog 63:1-2 2016

(C) 2016 by the American Dental Society of Anesthesiology
Before the use of these checklists, which have been borrowed from the aviation and nuclear power industries, anesthetists were already used to using these instruments. All comprehensively trained GA providers are used to using a preanesthetic checklist to ensure that all needed equipment, supplies, and drugs are available and that emergency suction is at hand. Before every anesthetic, I still mentally go through my SCOMLADI (suction, circuit, oxygen, machine check, laryngoscope, airway, drugs, IV) checklist for every case, multiple times a day before each and every anesthetic, even if there are 10 cases that day. MS. MAIDS (machine check; suction; monitor; airway; IV; drugs; special equipment, eg, shoulder roll; etc) is another popular preanesthetic checklist. Either checklist may be one of the most critical to use for any dental sedation/anesthesia provider.

We know that the majority of adverse sedation/GA events that occur in dental practice involve airway concerns. Anatomic upper airway obstruction, laryngospasm, and foreign body obstruction probably lead the list. Bronchospasm and aspiration round out the major common diagnoses, although the differential for adverse airway/pulmonary emergencies is vast. When seconds matter in the management of an airway emergency, having everything needed immediately at hand is very beneficial. Are properly sized oropharyngeal airways (OPAs) on your backstand or counter? These will reliably overcome upper-airway obstruction from the tongue when the head tilt/jaw thrust maneuver does not displace the tongue away from the posterior pharyngeal wall. This commonly happens when the patient enters a state of deep sedation/GA, even if the intent was moderate sedation. Of course, according to the definition of moderate sedation, no airway interventions, including the head tilt/jaw thrust maneuver, are required to maintain a patent airway. If the patient is making respiratory effort, use of the OPA may allow for unimpeded breathing. If this is not the case, the bagvalve-mask device or another positive-pressure ventilation (PPV) oxygen delivery system should be used. The diagnosis may be drug-induced hypopnea/apnea or laryngospasm. Is the PPV device attached to supplemental oxygen also immediately available in the operatory? Or will precious time be lost getting the E cylinder into the operatory as the pulse oximeter continues to decline? This could be a case of laryngospasm, which may respond to PPV. Without the OPA in place, however, we cannot be entirely sure if the tongue is still 
a source of obstruction, so all cases in which PPV is used for emergency care should likely have an OPA placed. If PPV is unsuccessful, GA providers will use succinylcholine and consider endotracheal intubation. Is succinylcholine immediately available in the operatory with needled syringes? Is intubation equipment close at hand? Moderate-sedation providers would consider sedation reversal with naloxone and/or flumazenil. Are these drugs immediately available in sufficient quantities with needled syringes in the operatory? For all providers, what is plan B? Are laryngeal masks or other supraglottic airways (eg, King LT-D, Combitube) immediately available? Transtracheal jet ventilation or even cricothyrotomy may be other options to allow for oxygenation while other interventions are undertaken to rescue the patient. Bronchospasm will respond to epinephrine, which should also be quickly available.

All sedation/GA providers should assess what equipment, drugs, and devices are appropriate for their individual practices. If you are not using checklists in your practice now, developing an emergency airway checklist is a good first step to help ensure that all items are present. Having these things immediately in the operatory during sedation serves many purposes. Having all equipment close at hand allows response time to be optimized. In addition, staff members who set up the equipment may come and go from the practice, so consistency in setup is more likely. Equally important is the fact that airway emergencies are relatively rare in dental practice. Many deep and moderate sedation providers may not routinely review emergency airway management as many anesthesiologists do who routinely render patients unconscious and apneic. When ventilation is compromised, having all the equipment close at hand allows the provider to immediately see what treatment options are available. And he or she will be more likely to avail themselves of those options. A written algorithm for managing this most common type of sedation emergency may also be of benefit to the entire team.

As checklists move from the hospital and ASC to the dental office, as they likely will, it will probably be sedation- and GA-trained dentists who will be at the forefront of bringing sensible policies and procedures to the dental office. In this way, the readers of Anesthesia Progress will hopefully make a profession-wide contribution to enhancing safety in dental practice more generally.

\section{REFERENCES}

Steven Ganzberg, DMD, MS Editor-in-Chief, Anesthesia Progress

1. World Health Organization. Safe surgery saves lives. Available at: http://www.who.int/patientsafety/safesurgery/ tools_resources/SSSL_Checklist_finalJun08.pdf. Accessed November 1, 2015.

2. Gawande A. A Checklist Manifesto. New York, NY: Henry Holt and Co; 2009. 